\title{
Twiddler's syndrome
}

\author{
Ramesh Dharawat ${ }^{1}$, Mohsen Saadat ${ }^{2}$
}

${ }^{1}$ San Joaquin General Hospital, Division of Cardiology, 500 W. Hospital Rd., French Camp, CA 95231, USA, ${ }^{2}$ San Joaquin General Hospital, Department of Internal Medicine, 500 W. Hospital Rd., French Camp, CA 95231, USA
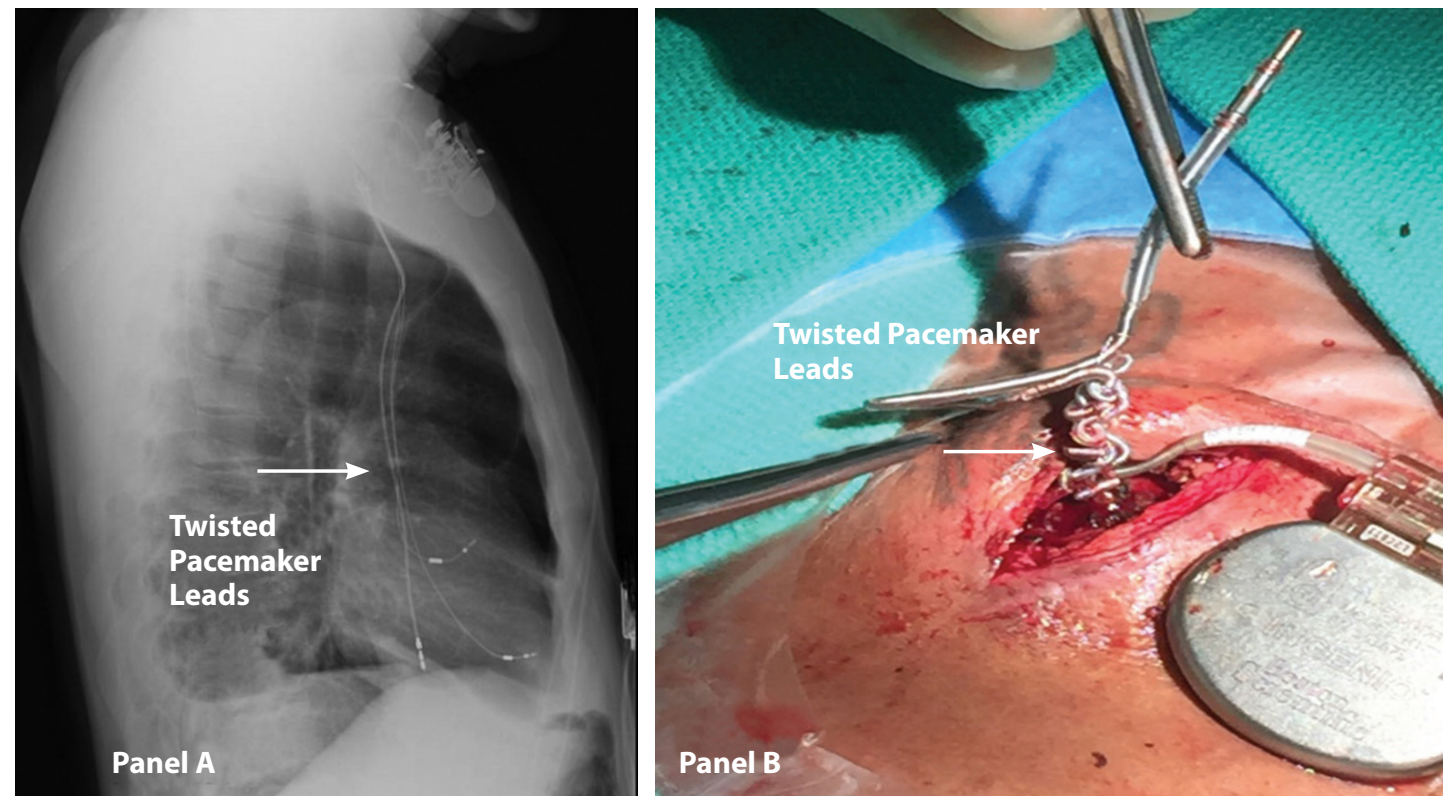

Twisted pacemaker leads or Twiddler's syndrome, are a rare cause of pacemaker malfunction. This condition typically results from the rotation of the device in its pocket, causing lead dislodgment (1). At risk individuals are usually obese, female, elderly, suffer from psychiatric disorders, or have a small size implanted device relative to the pocket size $(2,3)$. A 60 year old male with hypertension, Chronic Obstructive Pulmonary Disease, and Sick Sinus Syndrome presented to our emergency department with a complaint of chest pain which the patient described as an intermittent "firing/shock in my chest". Patient had a dual chamber pacemaker implanted in 2014, with new lead implantation two months previously due to the fracture of the right ventricular lead. Interrogation of the pacemaker revealed that the new lead was not sensing. Chest $\mathrm{x}$-ray showed dislodged ventricular lead in the right atrium (Panel A). The generator with severely twisted leads (Panel B) along with all the leads were extracted. New pacemaker 
leads were inserted on the right side using the right subclavian vein access and attached to the new generator. The patient is currently asymptomatic with regularly scheduled check up for the pacemaker.

Key words: Twiddler's syndrome - Sick sinus syndrome - Pacemaker - Pacing leads - Lead dislodgement.

Authors' contributions: Conception and design: RD and MS; Acquisition, analysis and interpretation of data: RD and MS; Drafting the article: RD and MS; Revising it critically for important intellectual content: RD and MS; Approved final version of the manuscript: $\mathrm{RD}$ and $\mathrm{MS}$.

Conflict of interest: The authors declare that they have no conflict of interest.
Correspondence:

mssaadat@sjgh.org

Tel.: + 209468 6043; Fax.: + 2094686246

Received: 28 September 2016

Accepted: 25 November 2016

\section{References}

1. Newland GM, Janz TG. Pacemaker-twiddler's syndrome: a rare cause of lead displacement and pacemaker malfunction. Ann Emerg Med. 1994;23(1):136-8.

2. Roberts JS, Wenger NK. Pacemaker twiddler's syndrome. Am J Cardiol. 1989;63(13):1013-6.

3. de Buileir M, Canver CC. Twiddler's syndrome complicating a transvenous defibrillator lead system. Chest. 1996;109(5):1391-4. 